\title{
Is dentistry the orphaned field of medicine? Ethical consideration for evidence-based dentistry
}

\author{
S. Sellars ${ }^{1}$ and H. S. Wassif*2
}

\section{Key points}

It was a watershed moment when Archie Cochrane published his work on Effectiveness and efficiency: random reflections on health services which was considered the start of evidence-based healthcare as we now know it. From there onward, evidence-based dentistry (EBD) has developed and evolved and there has been little attention to the interlink between EBD and ethics. The aim of this paper is to explore some of the ethical basis of evidence-based approach to healthcare utilising Beauchamp and Childress' four principles. EBD will be examined in relation to a variety of ethical theories. The case will be made that dentistry can, at times, be considered the orphaned field of medicine and an examination will be presented about how this may affect the uptake of EBD in practice. While exploring the strengths and weaknesses of EBD from an ethical viewpoint, we will highlight some of the challenges facing many dental practitioners in judging what is considered high-quality evidence and examine ways in which this could be improved with links to patient outcomes from an ethical perspective.

\section{Evidence-based dentistry}

Evidence-based dentistry (EBD) can be considered as an approach to dental practice which integrates a practitioner's expertise and the patient's needs and preferences with the latest and most relevant scientific evidence. ${ }^{1}$ It could be argued that EBD promotes both beneficence and patient autonomy, ${ }^{2}$ by the patient choosing and the practitioner being able to carry out the most clinically effective treatment. However, we believe that this somewhat oversimplifies the concept of EBD both clinically and from an ethical viewpoint for the reasons outlined in this article.

In Delivering better oral health: an evidence based toolkit for prevention, ${ }^{3}$ Public Health England's flagship document on preventative dentistry, only 48 recommendations

1Red Pepper Clinic, 131-133 Birchwood Avenue, Lincoln, LN6 0JE; ${ }^{2}$ University of Bedfordshire, Healthcare Practice, Putteridge Bury Campus, Hitchin Road, Luton, LU2 8LE. ${ }^{*}$ Correspondence to: Hoda S. Wassif

Email: hoda.wassif@beds.ac.uk

Refereed Paper. Accepted 19 November 2018 DOI: $10.1038 /$ sj.bdj.2019.145 of 113 (42\%) are based on strong evidence. The majority of Cochrane reviews ${ }^{4}$ also demonstrate that a large amount of evidence produced in the dental field is of low-quality with a moderate-to-high risk of bias.

We believe that this has clear implications to the beneficence and non-maleficence ${ }^{2}$ of EBD. If the quality and quantity of evidence is low, then any benefits of interventions are uncertain. This may lead to an unknown change in the risk-benefit balance of treatment and the treatment that is intended to benefit a patient may actually lead to harm.

It was argued that evidence-based practise may be more difficult in dentistry than medicine. ${ }^{5}$ This is partly because a broken tooth will not heal itself, as well as the nature of the dental industry and the flow of new and 'better' materials and techniques which, at times, tend to have short follow-up periods in clinical research which is often funded by the company themselves and therefore at risk of bias. It could also be argued that long-term randomised controlled trials of different treatment modalities or uses of different materials are not realistic. Because of this, much of the data collected comes from observational trials, and is subsequently at risk of being of lower value. From a utilitarian perspective, this may not matter, but biased evidence may be unreliable, and it could be argued that we have a duty to patients to ensure that the evidence collected is reliable. As it stands, practitioners are free to choose to perform treatments based on their own clinical expertise and training. It is possible, however, that if there is a move towards carrying out those treatments of which there is high quality evidence, there will be a significant ethical consequence.

\section{Patient and practitioner autonomy}

The uncertainty regarding evidence base may have a significant effect on both patient and practitioner autonomy. ${ }^{2,6}$ Practitioner autonomy is already somewhat restricted. For example, under the NHS GDS contract, only certain treatments are permitted to be carried out. This reduction in autonomy would be further apparent if only those treatments shown to have clear therapeutic effects were allowed to be performed.

If EBD standards are set too high, when the evidence base is low, practitioners will suffer 
loss of autonomy. This reduction in practitioner autonomy will then be passed onto the patient by fewer options for treatment being presented. However, fewer higher quality treatment options could be more beneficial than a larger number of lower quality options.

New and innovative treatment modalities may also have a lower level of evidence base, and therefore may be limited if only high evidence level treatments are allowed. This has the potential to stifle new research, and the subsequent improvement of the evidence base, in turn limiting the beneficial potential of treatments.

\section{Justice and the ethics of treatment funding}

The issue of justice comes into play when considering treatment funding. From a deontological viewpoint, practitioners have a duty to offer the best treatments to patients. Conversely the utilitarian view may be that the greatest good is served by treating a larger number of patients at a satisfactory level. From a virtue ethics ${ }^{2}$ perspective, it is virtuous to offer the best available treatment. Equally, a virtuous practitioner would want to treat as many people as possible with a satisfactory outcome. However, this raises more questions about what is considered 'satisfactory' and reinforces the argument for allowing more autonomy for experienced practitioners in making informed decisions in partnership with their patients.

Numerous organisations, including the Faculty of Dental Practice UK (FGDP) and Scottish Dental Clinical Effectiveness Programme (SDCEP), have produced evidence-based guidelines to aid in clinical practice. However, it was suggested that problems may arise when these guidelines are seen as laws to be followed. ${ }^{5}$ Instead, clinical guidelines could be upheld somewhere between laws and more generalist 'rules of thumb'. This prima facie approach to guidelines allows practitioner interpretation of the guidelines to suit individual patients. This in turn increases practitioner autonomy and beneficence of the treatment. In essence, this is true EBD, balancing current scientific evidence with clinical knowledge and patient needs. However, this approach requires caution as deviating from accepted evidence-based standards of care may be seen as not giving patients the best care available. If the best intervention is largely agreed across the profession, then not following this line of treatment can be seen as unethical. However, will following strict guidelines stifle progress, innovation and questioning those guidelines? For example, the use of rubber dam in endodontic procedures is considered a basic standard of care, from patient safety (non-maleficence) and treatment success (beneficence) viewpoint. ${ }^{2}$ However, the patient may want to express their autonomy by requesting the treatment without rubber dam, but an ethical practitioner should deny this request. In this case, paternalism rules and the beneficence (or non-maleficence) of using rubber dam precludes the option of treatment without.

\section{Dentistry, an orphaned field of medicine?}

It could be argued that any treatment modality or intervention without good evidence is ethically unacceptable. ${ }^{7}$ This is of particular relevance to those fields of medicine where research is considered weak, low quality, and lacking in financial incentive. The claim is that the difficulties of obtaining high quality research groups have a number of knock-on ethical effects. The difficulty in obtaining meaningful information from research would potentially lead to less research funding. This is an example of unjust distribution of healthcare resources, which again would lead to lower patient autonomy, as potential beneficial treatment modalities lay undiscovered or unevaluated. In some 'orphaned' fields, the ethical issues arising are often related to decision-making and patient-professional relationships. Expanding the depth of evidence available will help these discussions have more certain outcomes.

The formulation and introduction of evidence-based guidelines into practice should, theoretically, help improve patient outcomes. However, dentists often mistrust the results of academic research ${ }^{8}$ and tend to trust their own clinical experience over clinical trials.

This view of 'it works in my hands' is one of the biggest stumbling blocks to the acceptance of EBD into routine practice. It ignores any cognitive biases which affect how we judge our own experiences. Both confirmation bias ${ }^{9}$ and the Dunning-Kruger effect ${ }^{10}$ can affect practitioners' perception of their own skills and the success or failure of their treatment. Practitioners need to be aware of the biases which may influence their decision making. Without this skill, both treatment choices and treatment outcomes can be compromised.

Additionally, in order for dentists to take a more evidence-based approach to clinical practice, clinical examples should be given alongside raw statistics. ${ }^{8}$ This increases the uptake of new evidence-based techniques and allows a more beneficent profession as a result of it. There is a risk that dentists may not keep up to date with current accepted protocols for treatment and regularly updated standard operating procedures may act as a remedy to this. ${ }^{11}$ The ethical impact of this proposal would be complicated. On the one hand, the implementation of a best-practice standard would have the potential to increase benefit and reduce harm. It would also help identify areas where research and evidence base is lacking, allowing more focused and beneficial research to be carried out. The suggestion may limit practitioner autonomy and/or patient autonomy as discussed earlier, especially in areas where multiple techniques or approaches are acceptable.

\section{The media and evidence}

The media portrayal of experts and evidence is likely to affect patient acceptance of evidencebased dentistry. This is mainly due to the effect of the media on personal choices. Again, this is likely to affect practitioner autonomy, as patients may be inclined to request treatments which they perceive to be popular.

An item on ITV's 'This Morning' programme, ${ }^{12}$ for example, was promoting natural ways to whiten teeth and showed interventions such as oil pulling with coconut oil and cleaning teeth with a bicarbonate of soda solution. These interventions have little to no evidence to support them, but are likely to affect viewers' perception of what is considered a valid treatment. Recommending the use of abrasive substances such as bicarbonate of soda on teeth could be harmful and could be argued as unethical. This is not only because of the potential for harm, but because it may give credence to further evidence-free claims that similar practitioners may be making which in themselves may be harmful.

Conversely, practitioners such as 'The Singing Dentist ${ }^{\prime 13}$ help promote dentists and dentistry as an open, caring and fun sector of healthcare. This is likely to encourage people to visit the dentist and has potential to improve overall dental health. This ties into the General Dental Council remit to maintain and promote public confidence in the profession. ${ }^{14}$ 
Print media also has a role to play in patient perception of evidence with some newspapers misreporting or exaggerating research studies. This can lead to a mistrust of dentists who may be viewed as sending out mixed messages, or worse, not knowing which action is correct.

\section{Improving uptake and relevance of evidence-based dentistry}

In 2004, a case for embracing the uncertainty of available evidence was presented ${ }^{15}$ outlining that the dichotomisation of science, and related healthcare, into 'proven' and 'unproven' can have serious consequences, both practically and ethically. The very nature of scientific research dictates that current knowledge is constantly updated and so our implementation of that science needs to be continually changing.

The importance of evidence-based healthcare can be seen most starkly in the CRASH study ${ }^{16}$ regarding the treatment of head injury with corticosteroids. Before the results of this study it was assumed that patients with a head injury should be given steroids to reduce swelling of the brain. The CRASH trial showed that, in fact, giving steroids was actively harmful to patients. It was calculated that around 10,000 people died due to the false assumption that steroids would help rather than harm. Although it is unlikely that there would be any similar consequences regarding any form of dental treatment, we can't be sure that our practices are benefiting patients unless we study our actions. Similarly, what may have been considered beneficial in the past may eventually be seen as harmful. For example, the current trend towards phasing down of the use of amalgam after it stayed in use routinely for over 150 years. With the realisation of the environmental impact of mercury and the change in patient perception of safety, amalgam is falling out of favour.

One suggestion could be the introduction of the 'reader ${ }^{17}$ who would collate and present the best evidence available to practitioners. This already takes place in other fields of medicine and would be relatively simple to implement. Ethically, this would be just, as each practitioner would be in receipt of the same evidence and guidelines. It would also help increase treatment success and therefore the beneficence of the profession as a whole.

\section{Conclusion}

It could be argued that within dentistry, and in comparison to medicine, there is an evidence based void. A large amount of research is being concentrated on complex procedures, such as implant placement success rate. In comparison, there is less research going into basic dentistry such as tooth brushing technique and cavity design. We believe that this raises many ethical questions as practitioners are unable to provide a truly beneficent service to patients if there is no good evidence to show that the treatment offered is truly beneficial. Similarly, patient autonomy is affected. If there is no way to know which treatment option is shown to be most effective, then a patient cannot make a fully informed decision. From a funding point of view, money may be being wasted on ineffective treatments but there is no way to know without good quality evidence. In a system where funding often comes from a limited government budget, this can create an uncertainty in equality; where some patients may be receiving more expensive but less effective treatment.

Limiting the use of efficient but superseded practices may be beneficial to patients. This would, though, require the profession to train in new and updated practices. Although this may be expensive and logistically taxing, it is one way of ensuring that effective treatment which has been proven to benefit patients is being fully implemented. Focusing on the effectiveness and the importance of EBD is paramount at all levels of training and education, starting from undergraduate dental students and throughout all aspects of continuous professional development for experienced practitioners.

EBD is without doubt of overall benefit to the profession and patients in general. We believe that EBD is key to delivering success to both practitioners and patients by choosing treatment options that are proven to be effective and thus reducing harm to patients and increasing patients trust in the profession.
Striving to improve the quality of evidence available for all, should be one of the main priorities of the dental profession. This can be done by implementing the role of professional reader to consolidate available evidence and make access simpler. Practice-based research should be encouraged and well-funded. Emphasis on EBD should be an integral part of the undergraduate curriculum as well as training on how to conduct and interpret clinical trials to ensure that dentistry is never viewed as an orphan branch of medicine.

1. ADA Centre for Evidence Based Dentistry. About EBD. 2019. Available at: https://ebd.ada.org/en/about (accessed January 2019).

2. Beauchamp T L, Childress J F (eds). Principles of Biomedical Ethics. 7th ed. Oxford: Oxford University Press, 2013.

3. Public Health England. Delivering better oral health: an evidence-based toolkit for prevention. 3rd ed. 2017. Available at: https://assets.publishing.service.gov.uk/ government/uploads/system/uploads/attachment_data/ file/605266/Delivering_better_oral_health.pdf (accessed January 2019).

4. Cochrane Library. 2019. Available at: https://www. cochranelibrary.com/ (accessed January 2019).

5. Espelid I, Alanen P, Hofmann B. Evidence based dentistry and ethics. Den Norske Tannlegeforenings Tidende 2013; 1: 28-32.

6. Cannavina C D, Cannavina G, Walsh T F. Effects of evidence-based treatment and consent on professional autonomy. Br Dent J 2000; 188: 302-306.

7. Vos R, Willems D, Houtepen R. Coorinating the norms and values of medical research, medical practice and patient worlds - the ethics of evidence based medicine in orphaned fields of medicine. J Med Ethics 2004; 30: 166-170.

8. Sbaraini A, Carter S M, Evans R W. How do dentists understand evidence and adopt it in practice? Health Educ J 2011; 71: 195-204.

9. Eli I. Reducing confirmation bias in clinical decision-mak ing. J Dent Educ 1996; 60: 831-835.

10. Kruger J, Dunning D. Unskilled and unaware of it: how difficulties in recognizing one's own incompetence lead to inflated self-assessments. J Pers Soc Psychol 1999; 77: 1121-1134.

11. Faggion C M Jr, Tu Y K. Standard operating procedures approach for the implementation of the evidence-based dentistry concept in dental practice. J Evid Based Dent Pract 2007; 7: 102-107.

12. ITV. Nine natural ways to whiten your teeth. 2017. Available at: http://www.itv.com/thismorning/style-beauty/ nine-natural-ways-to-whiten-your-teeth (accessed January 2019).

13. The Singing Dentist. 2019. Available at: http://singingdentist.com/ (accessed January 2019).

14. General Dental Council. Standards for the Dental Team. 2013. Available at: https://www.gdc-uk.org/api/files/ NEW\%20Standards\%20for\%20the\%20Dental\%20 Team.pdf (accessed January 2019).

15. Vineis P. Evidence-based medicine and ethics: a practical approach. J Med Ethics 2004; 30: 126-130.

16. Czekajlo M S, Milbrandt E B. Corticosteroids increased short and long-term mortality in adults with traumatic head injury. Crit Care 2005; 9: E21.

17. Chiappelli F, Prolo P. The meta-construct of evidence-based dentistry. Part 2. J Evid Base Dent Pract 2002; $2: 1-7$ 